\title{
A 60-year-old woman with recurrent cellulitis
}

\author{
Rohit Vijh BSc, Rupal Shah MD MHPE, Nisha Andany MD MPH
}

Cite as: CMAJ 2019 March 4;191:E257-9. doi: 10.1503/cmaj.180686

A 60 -year-old woman with diabetes and venous insufficiency presents with 5 days of pain in her left leg. In the past 3 years, she has had 4 episodes of cellulitis, most recently 5 months ago. On examination, she is afebrile and hemodynamically stable. The patient's body mass index (BMI) is $35 \mathrm{~kg} / \mathrm{m}^{2}$ (obesity class). Her left leg is warm, erythematous, swollen and tender to palpation, with maceration between the toes, but no ulceration, crepitus or purulence. D-dimer testing is negative. She asks about antibiotic prophylaxis for recurrent cellulitis.

\section{What further questions should be asked on history?}

The provided history is strongly suggestive of cellulitis, an acute infection that typically affects the lower extremities in a unilateral distribution. ${ }^{1}$ Further history should rule out alternative diagnoses such as venous stasis, volume overload and deep vein thrombosis. ${ }^{1}$ The patient should also be evaluated for underlying conditions that predispose to nonpurulent cellulitis (Box 1). The annual risk of recurrence after a single episode of cellulitis is $8 \%-20 \% ;^{3}$ thus, her history poses a substantial risk. Additionally, her underlying venous insufficiency may impair blood flow and wound healing, further increasing risk of recurrent infection. ${ }^{2,3}$ Assessment of diabetes control is important to cardiovascular health; however, in the absence of skin ulceration, diabetes has not consistently been shown to increase risk of nonpurulent cellulitis. $^{2}$

\section{What findings from the physical examination support this diagnosis?}

On physical examination, this patient presents with 4 cardinal features of cellulitis: erythema (with poorly demarcated borders), swelling, warmth and tenderness to palpation. ${ }^{1}$ The absence of bilateral extremity involvement reduces the likelihood of alternative diagnoses including stasis dermatitis or volume overload. ${ }^{1}$ Cellulitis and deep vein thrombosis can be difficult to differentiate. A clinical prediction rule (Wells criteria for deep vein thrombosis score), combining historical and physical examination findings, can be used to determine the probability of deep vein thrombosis. ${ }^{4}$ Her Wells score is 0 , based on the results of her physical examination, lack of thrombotic risk factors and cellulitis being as likely a diagnosis. Focused physical examination should also assess for modifiable risk factors for recurrent cellulitis, including predisposing comorbid conditions and those that compromise skin integrity (Box 1$).{ }^{2}$ In this case, elevated BMI, venous insufficiency and maceration between the toes, suggestive of tinea pedis, all increase her risk of subsequent episodes. ${ }^{1,3}$

\section{Should further investigations be ordered?}

Cellulitis is a clinical diagnosis. ${ }^{1}$ Investigations for alternative diagnoses, including noninfectious causes, should be considered in all cases, but particularly if therapy with appropriate antibiotics is ineffective. ${ }^{1}$ This patient's Wells score indicates a low pretest probability of deep vein thrombosis; combined with negative D-dimer testing, deep vein thrombosis is reliably excluded without the need for confirmatory ultrasound. ${ }^{4}$ Although the history and physical examination in this patient are strongly suggestive of cellulitis, warranting no further confirmatory tests, investigations for relevant risk factors should be considered.

\section{How should this case be managed acutely?}

Acute management of cellulitis is guided by the most likely causative organism(s) and severity of presentation. $\beta$-Hemolytic streptococci (predominantly Streptococcus pyogenes) and methicillin-susceptible Staphylococcus aureus account for the majority of cases, but microbiology can be altered with penetrating trauma, immunosuppression and water exposure. ${ }^{1}$ Methicillin-resistant S. aureus (MRSA) is an uncommon cause of nonpurulent cellulitis, and empiric treatment is generally not required; however, MRSA should be considered in cases of purulent cellulitis, particularly in those with established risk factors. ${ }^{1,3}$ Severity of nonpurulent cellulitis can range from mild infection, with no systemic involvement, to severe sepsis and shock. ${ }^{1}$ Given this patient's clinical stability, oral antibiotic therapy can be prescribed on an outpatient basis. A first-generation cephalosporin targets the most likely organisms. ${ }^{3}$ In addition, topical antifungal therapy and wound care may be used to address the patient's tinea pedis. $^{3}$

\section{Should this patient receive antibiotic prophylaxis?}

This patient should not receive antibiotic prophylaxis at this time. As per the Infectious Diseases Society of America guideline, prevention of recurrent cellulitis first involves identifying and managing reversible predisposing factors. ${ }^{3}$ Strategies pertinent to this case may include weight loss, proper foot hygiene, treatment of tinea pedis and compression therapy for venous 
Box 1: Risk factors for nonpurulent leg cellulitis ${ }^{2}$

\begin{tabular}{|c|c|c|}
\hline Risk factor & $\mathrm{OR}^{\star}(95 \% \mathrm{CI})$ & Treatment \\
\hline \multicolumn{3}{|l|}{ Local risk factors } \\
\hline History of cellulitis & $40.30(22.59-71.90)$ & NA \\
\hline $\begin{array}{l}\text { Lymphedema or chronic leg } \\
\text { edema }\end{array}$ & $6.77(3.46-13.27)$ & $\begin{array}{l}\text { Treatment of underlying cause (e.g., compression therapy for } \\
\text { venous insufficiency, diuretics, water and salt restriction for } \\
\text { heart failure) }\end{array}$ \\
\hline $\begin{array}{l}\text { Cutaneous barrier disruption } \\
\text { (skin trauma or wound }<1 \\
\text { mo duration) }\end{array}$ & $19.11(9.13-39.98)$ & $\begin{array}{l}\text { Treatment with an antimicrobial agent } \\
\text { Harm reduction with safe and clean injection sites and } \\
\text { equipment }\end{array}$ \\
\hline Current leg ulcer & $13.65(7.89-23.59)$ & Wound care with or without surgical débridement \\
\hline Tinea pedis & $3.16(1.9-5.25)$ & Antifungal agents \\
\hline $\begin{array}{l}\text { Excoriating skin disease } \\
\text { (e.g., eczema, psoriasis) }\end{array}$ & $4.37(2.7-7.08)$ & Treat underlying cause (e.g., topical corticosteroids) \\
\hline Previous history of ulcer & $4.47(1.6-12.51)$ & NA \\
\hline Previous leg surgery & $2.26(1.71-4.12)$ & NA \\
\hline \multicolumn{3}{|l|}{ General risk factors } \\
\hline Overweight (BMI > 25) & $1.87(1.26-2.79)$ & Weight reduction strategies (dietary and exercise regimens) \\
\hline Obesity (BMI > 30) & $2.37(1.39-4.05)$ & Weight reduction strategies (dietary and exercise regimens) \\
\hline
\end{tabular}

insufficiency (Box 1). ${ }^{3}$ Reminders from clinicians can improve adherence to compression therapy, based on the results of one 4-week randomized controlled trial (RCT). ${ }^{5}$ Referral for home support services may also improve adherence to nonpharmacologic strategies.

Chronic antimicrobial prophylaxis is an additional strategy that may be appropriate for certain patients with recurrent cellulitis. The efficacy of antimicrobial prophylaxis in preventing recurrent cellulitis is supported by results from 3 RCTs. ${ }^{6-8}$ The largest and most recent RCT showed that in individuals with 2 or more episodes in a 3-year period, chronic therapy with oral penicillin $\mathrm{V}$ potassium delayed time to recurrence by 94 days, and reduced risk of recurrence by $45 \%$ while on treatment. ${ }^{7}$

However, consideration of this strategy requires careful assessment of the risks and benefits. A systematic review of trials notes that these RCTs do not adequately address whether modifiable risks factors were optimized; nor do they provide sufficient data on important safety outcomes such as antimicrobial resistance and Clostridium difficile infection. ${ }^{9}$ No studies have directly compared efficacy of risk factor modification versus antimicrobial prophylaxis versus combination strategies. Given these limitations, the current Infectious Diseases Society of America guideline suggests prophylaxis be reserved for patients who, despite appropriate optimization of risk factors, continue to have 3 to 4 infections per year. ${ }^{3}$ At that point, referral to an infectious diseases specialist for discussion of prophylaxis may be considered. For individuals with less frequent recurrences, expert opinion suggests episodic treatment with a limited course of antibiotics. ${ }^{3}$ Options for prophylaxis include penicillin or macrolides, for a duration of up to 1 year, after which indications for ongoing pro- phylaxis should be reassessed., ${ }^{3,10}$ Notably, benefits of antibiotic prophylaxis are lost once therapy is stopped. ${ }^{7,10}$

\section{Case revisited}

This patient's history and examination were consistent with cellulitis. She was successfully treated with 5 days of oral cephalexin. She had several modifiable risk factors that were addressed: weight loss was encouraged, and her tinea pedis was treated with a topical antifungal agent. She was prescribed compression stockings to manage venous insufficiency, but could tolerate compression for only short periods of time.

She should be counselled on signs and symptoms of cellulitis that would prompt medical attention. She had a subsequent episode of cellulitis 18 months later, again responsive to cephalexin. Although she remains at risk of recurrent cellulitis, the frequency of episodes has improved with modification of risk factors and does not warrant chronic antibiotic prophylaxis at this time.

\section{References}

1. Raff AB, Kroshinsky D. Cellulitis: a review. JAMA 2016;316:325-37.

2. Quirke M, Ayoub F, McCabe A, et al. Risk factors for nonpurulent leg cellulitis: a systematic review and meta-analysis. Br J Dermatol 2017;177:382-94.

3. Stevens DL, Bisno AL, Chambers HF, et al.; Infectious Diseases Society of America. Practice guidelines for the diagnosis and management of skin and soft tissue infections: 2014 update by the Infectious Diseases Society of America. Clin Infect Dis 2014;59:e10-52.

4. Wells PS, Owen C, Doucette $\mathrm{S}$, et al. Does this patient have deep vein thrombosis? JAMA 2006;295:199-207.

5. Uhl JF, Benigni JP, Chahim M, et al. Prospective randomized controlled study of patient compliance in using a compression stocking: importance of recommendations of the practitioner as a factor for better compliance. Phlebology 2018;33:36-43. 
6. Kremer M, Zuckerman R, Avraham Z, et al. Long-term antimicrobial therapy in the prevention of recurrent soft-tissue infections. J Infect 1991;22:37-40.

7. Thomas KS, Crook AM, Nunn AJ, et al.; U.K. Dermatology Clinical Trials Network's PATCH I Trial Team. Penicillin to prevent recurrent leg cellulitis. N Engl J Med 2013;368:1695-703.

8. Sjöblom AC, Eriksson B, Jorup-Rönström C, et al. Antibiotic prophylaxis in recurrent erysipelas. Infection 1993;21:390-3.

9. Dalal A, Eskin-Schwartz M, Mimouni D, et al. Interventions for the prevention of recurrent erysipelas and cellulitis. Cochrane Database Syst Rev 2017;6:CD009758.

10. Oh CC, Ko HC, Lee HY, et al. Antibiotic prophylaxis for preventing recurrent cellulitis: a systematic review and meta-analysis. J Infect 2014;69:26-34.
Decisions is a series that focuses on practical evidence-based approaches to common presentations in primary care. The articles address key decisions that a clinician may encounter during initial assessment. The information presented can usually be covered in a typical primary care appointment. Articles should be no longer than 650 words, may include one box, figure or table and should begin with a very brief description (75 words or less) of the clinical situation. The decisions addressed should be presented in the form of questions. A box providing helpful resources for the patient or physician is encouraged.

\section{Competing interests: None declared.}

This article has been peer reviewed.

The clinical scenario is fictional.

Affiliations: Faculty of Medicine (Vijh) and Department of Medicine (Shah, Andany), University of Toronto; Division of General Internal Medicine (Shah), Toronto Western Hospital; Division of Infectious Diseases (Andany), Sunnybrook Health Sciences Centre, Toronto, Ont.
Contributors: All of the authors contributed to the conception and design of the work, drafted the manuscript, revised it critically for important intellectual content, gave final approval of the version to be published and agreed to be accountable for all aspects of the work.

Acknowledgements: The authors thank Drs. Emily Siu and David Makary for reviewing this paper and providing valuable feedback and suggestions.

Correspondence to: Nisha Andany, Nisha.Andany@sunnybrook.ca 